\title{
Sensor-OSTR: Novel energy-efficient dynamic TDMA frame size-based MAC Protocol for Wireless Multi-hop Sensor Networks
}

\author{
Afef Sayadi \\ Télécom and Management SudParis \\ 9 Rue Charles Fourier 91000 \\ Evry, France \\ afef.sayadi@it-sudparis.eu
}

\author{
Saoucene Mahfoudh \\ Télécom and Management SudParis \\ 9 Rue Charles Fourier 91000 \\ Evry, France \\ Saoucene.Ridene@it-sudparis.eu
}

\author{
Anis Laouiti \\ Télécom and Management SudParis \\ 9 Rue Charles Fourier 91000 \\ Evry, France \\ anis.laouiti@it-sudparis.eu
}

\begin{abstract}
Wireless sensor networks are one of the key technologies to ensure ubiquitous connectivity for the internet of future where billions of things must be connected. As sensor nodes are generally energy-constrained, the critical issue to face is how to reduce energy consumption so that the network life is prolonged. Time Division Multiple Access based protocols are inherently energy-efficient since nodes are awake only during their slots and sleep the rest of the time. To our knowledge, few are the works that have investigated the impact of a dynamic TDMA frame size on the network energy consumption. In this paper, we propose a new energy efficient MAC protocol for wireless sensor networks, called Sensor One shot Slot TDMAbased Reservation (S-OSTR). S-OSTR brings the idea of a dynamic active period, since it deploys a dynamic frame size that is built slot-by-slot according to nodes arrival to the network leading to a shorter achieved frame size. The short frame size combined with an appropriate spatial reuse scheme contributes to significant save of energy. The S-OSTR energy efficiency has been approved by a set of simulations comparing our proposal with SERENA [1].
\end{abstract}

Keywords- sensor networks; dynamic frame; TDMA; node activity scheduling; energy efficiency.

\section{INTRODUCTION}

A wireless sensor network is a set of nodes (sensors) deployed over a geographical area to ensure short-range device communications (e.g. smart home) and to monitor physical phenomena such as humidity, temperature and so on. An energy-efficient MAC protocol is of primary concern in wireless sensor networks. Such networks, indeed, are energyconstrained, since they need to fulfill a long lifetime; while sensor batteries are power-limited. How to reduce the energy consumption is, then, a critical issue to face in wireless sensor networks. In [2], five major causes of energy waste are identified: collision, overhearing, control packet overhead, idle listening, and over emitting. Beyond these observations, a good strategy to save more energy in sensor networks needs to: (1) minimize the waste of energy due to the aforementioned causes, and (2) prolong the periods during which nodes are in the sleep state.

Node activity scheduling is an energy efficient strategy that combines these two attributes [3]. Several MAC protocols based on node activity scheduling have been proposed in the literature. They are mainly classified in three groups: contention-based, TDMA-based, and hybrid protocols. The contention-based solutions are the most popular class. They integrate a sleep/wakeup scheme in the channel access functionalities. [4], [5], and [6] are some examples of these protocols. TDMA-based protocols [7], [8], and [9] give a natural advantage regarding the energy efficiency, since the transmissions are scheduled and collisions are avoided leading to energy savings. Hybrid MAC protocols take advantage from both the TDMA access scheme and the CSMA access scheme. One of the most interesting works among this class is Zebra MAC (Z-MAC) [10]. Z-MAC specifies that nodes should be awake during the whole active period. This contributes to additional waste of energy, since a node should only be awake when it is in transmit or receive state. Based on this observation, [1] proposes the SchEduling RoutEr Nodes Activity (SERENA) protocol. SERENA uses a distributed and localized two-hop coloring algorithm for slot allocation.

In this paper, we propose a new MAC protocol for wireless sensor networks called Sensor One shot Slot TDMA-based Reservation (S-OSTR) MAC protocol. S-OSTR prolongs the network lifetime based on three major features: (1) scheduling the transmissions based on a TDMA channel access scheme. (2) Providing a dynamic and short active period thanks to its slot-by-slot frame size increase, leading to a shorter achieved frame size. (3) Coordinating the active and sleep periods such that a node is awake only during its own slot and the slots assigned to its one-hop neighbors.

The remainder of the paper is organized as follows. In Section II, a detailed description of S-OSTR is presented. The S-OSTR performances evaluations are held in Section III. Finally, we conclude this paper in Section IV.

\section{S-OSTR MAC PROTOCOL}

\section{A. S-OSTR Overview}

S-OSTR is a distributed medium access protocol for wireless multi-hop sensor networks that schedules transmissions in a slotted environment. With S-OSTR, time is divided in periodic polling cycles. The duration of each polling cycle is assumed to be fixed and defined by the application running on top of the S-OSTR protocol. For a 
coherent work of the network, sensors are required to be perfectly synchronized in order to wake up in the beginning of each polling cycle at the same time. Clock synchronization in wireless environment, and in particular in sensor networks is a critical issue to face. However, it is beyond the scope of our work. [14] gives detailed surveys on time synchronization techniques.

Each polling cycle is composed of two periods of time: an active period where DATA packets are transmitted and an inactive (sleep) period where all sensors turn off their radio to save energy. Intuitively, the longer the inactivity period is the more energy-efficient is the MAC protocol. Hence, S-OSTR uses a dynamic active period that can adapt to network topology changes. The active period consists on one TDMA frame cycle that is built slot-by-slot according to nodes arrival/departure to/from the network. The frame is divided into two parts: a CONTROL sub-frame where the channel access is contention-based and a DATA sub-frame where the channel access is scheduled-based. Since we are mainly interested in providing an energy-efficient solution, rather than a traffic rate adaptive solution, we make the assumption that each node only reserves one time slot per frame. We also assume that the CONTROL sub-frame consists of the first time slot of each frame.

\section{B. S-OSTR: Protocol Description}

The S-OSTR MAC protocol aims to save energy by reducing the active period in the polling cycle. To this end, four features are deployed: (1) First, the S-OSTR TDMA channel access technique gives a natural advantage of energy conservation because of the periodicity of the TDMA frame. (2) Due to the S-OSTR time slot allocation algorithm, a shorter frame is achieved, so that active period is reduced (3) Moreover, S-OSTR uses an alternate between a dynamic active period and a dynamic sleep period in one polling cycle. When the dynamic active period relative to one sensor comes to the end, it turns off its radio and enters the sleep period. (4) Finally, S-OSTR does not require nodes to be awake during the whole active period, but only during the CONTROL subframe, during their own assigned slot, and during the slots assigned to their one-hop neighbors.

The initialization period of a new incoming node is named the sensing period. During this period, the node senses the channel to gather the HELLO packets exchanged periodically by all nodes in the network. The HELLO packets incur the frame size, and the assigned time slot of the sender as well as its one-hop neighbors. After the sensing period, the new node maintains information about the slots distribution and the frame sizes in its contention area enabling it to properly choose a time slot based on the S-OSTR slot selection algorithm: With S-OSTR, a new node first selects the maximum frame size denoted by $K$, among all the frame sizes in its two-hop neighborhood. If at least one time slot is free among this maximum frame size, the new node chooses the slot with the lowest number. Otherwise, it chooses the slot number $(K+1)$. A slot is considered a free slot for a node $n$ if it is not assigned to any node in node $n$ two-hop neighborhood.
Once the slot is chosen, the new node sends a request packet during the CONTROL sub-frame to request for the intended slot reservation. It also launches a timer to avoid waiting for the REQ confirmation indefinitely. Upon the REPLY receipt, the new node stops the timer. In case the timer comes to the end, the new node initiates a new reservation process in the next polling cycle.

\section{a) Slot Conflict Resolution in S-OSTR}

In S-OSTR a conflict of slot assignment occurs in two cases: either when a new node connects to two or more nodes to which the same slot is assigned, or when a node moves such that it enters the contention area of another node assigned the same slot number. In the former case, while joining the network, a new node verifies whether two of its one-hop neighbors have the same assigned slot. If it is the case, the node delays its reservation, and sends a conflict (CONF) packet including the number of the slot causing the conflict. Upon the receipt of the CONF packet, the conflicting node releases its slot and enters a new reservation phase in the next polling cycle. Meanwhile, the new node that has detected the conflict increases its contention window size and contends for the channel access to enter the reservation phase once again. In the second case, the periodic HELLO packet exchange allows both conflicting nodes to update their neighborhood table. While checking their tables, they both detect the conflict between their own assigned slot and the slot attributed to one of their neighbors. Consequently, S-OSTR specifies that the one with the highest MAC address releases its slot and enters a new reservation phase.

\section{S-OSTR PERFORMANCES EVALUATION}

To evaluate the performances of S-OSTR, we run multiple sets of simulations for different wireless networks using OMNET++ [11] simulator. We consider a multi-hop environment with random generated topologies where the number of nodes varies from 50 to 150 nodes. The measurements are performed based on User Datagram Protocol (UDP) traffic. UDP traffic is sent at Constant Bit Rate (CBR). The channel has a physical data rate of 2 Mbps. As we are interested in MAC layer performances, a static routing is used to define 20 user traffic flows, with randomly chosen sources and destinations. The polling cycle is fixed to 50 time slots, where the duration of one time slot is set to $5 \mathrm{~ms}$. Simulation results are averaged over 10 simulation runs. In order to study the energy efficiency of the S-OSTR protocol, we have included the battery package in the simulation. This package is part of the mobility framework project $[12,13]$.

\section{A. S-OSTR: Slot Reuse}

The S-OSTR protocol deploys a slot assignment mechanism that favors slot reutilization. To highlight the SOSTR spatial reuse feature, we consider a network of 150 nodes, achieving a frame size equal to 21 slots (including slot 0 dedicated to the CONTROL sub-frame). Fig.1. provides the slot reuse achieved with S-OSTR for this configuration. It provides the distribution of shared slots among the nodes in the network. We first notice, that all slots are used, and there is 
no empty slot. In this configuration, the distribution of shared slots is as follow: 5 slots are shared by 7 nodes, 3 slots are reused by 8 nodes, $30(5+6+9+10)$ nodes share 2 slots, and 31 nodes reuse one slot. This reflects the effectiveness of the slotby-slot frame size increase, and its robustness against the waste of energy due to free slots appearance.

\section{B. S-OSTR Evaluation with comparison to SERENA [1] 1) Simulation environment}

To demonstrate the effectiveness of our proposal, we compare its performances with that of the two-hop coloring algorithm deployed in SERENA. For fair comparison sake, we compare SERENA coloring algorithm with a special version of S-OSTR denoted by: S-OSTR without HELLO mechanism. First, S-OSTR is running with HELLO mechanism turned on in order to enable the nodes join the network until the intended number of nodes is reached. We refer to this phase as the network setting phase. It is to mention that the energy consumed during this phase is not taken into account. Once the network setting phase is over, nodes disable the HELLO mechanism and run the S-OSTR without HELLO mechanism protocol. Notice that this set of simulations is based on the same simulations parameters described in the beginning of Section III for both S-OSTR (with and without Hello mechanism) and SERENA.

2) Network lifetime and user data delivered

In Fig.2, we plotted the achieved network lifetime for both versions of S-OSTR (enabling and disabling the Hello mechanism) and for SERENA for a number of nodes in the network rising from 50 to 150 nodes. The results highlight the capacity of S-OSTR to achieve higher network lifetime comparing to SERENA even though the HELLO mechanism is enabled. In Fig.3, the amount of user data delivered with SOSTR and SERENA is plotted. The results go hand in hand with the network lifetime achieved with both protocols.

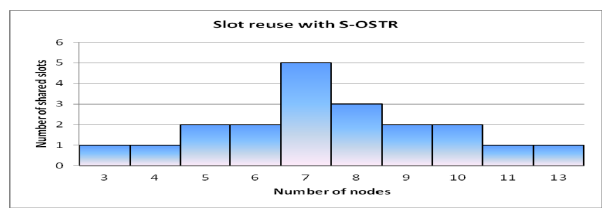

Figure $1:$ Slot reuse with S-OSTR

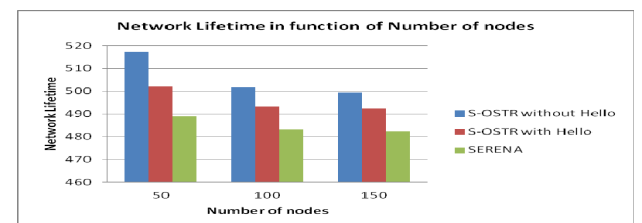

Figure 2 : Network lifetime comparison between S-OSTR (with and without Hello mechanism) and SERENA.

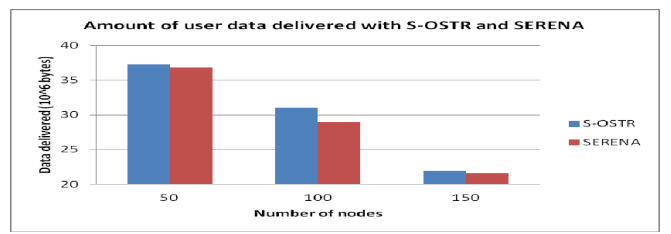

Figure 3 : Amount of user data delivered with S-OSTR and SERENA

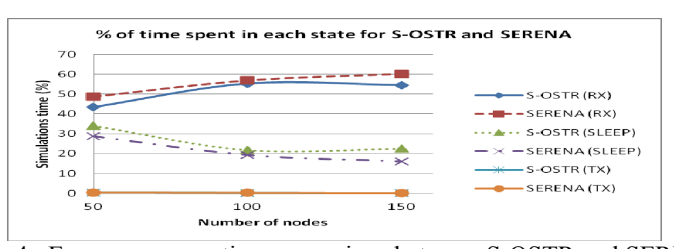

Figure 4 : Energy consumption comparison between S-OSTR and SERENA

\section{3)Distribution of node energy consumption}

In this Section, the distribution of energy consumption is analyzed. Fig. 4 represents the rate of time spent in TX (Transmit), RX (Receive), and SLEEP states, for S-OSTR and SERENA. The results show that with S-OSTR, nodes spend less time in TX and RX states comparing to SERENA for different number of nodes. Consequently, S-OSTR spares more energy comparing to SERENA since it increases the amount of time spent in the SLEEP state.

\section{CONCLUSION}

In this paper, we have proposed a new energy-efficient MAC protocol for wireless sensor networks called Sensor OSTR (S-OSTR). S-OSTR brings the idea of a dynamic active period that quickly accommodates to network changes and ensures a shorter frame size, i.e. shorter active period. As enhancement of S-OSTR, we intend to adopt a variable length for time slots. The length of each time slot will be deduced based on the interference level which is proportional to the reuse rate of the slot.

\section{REFERENCES}

[1] P. Minet, S. Mahfoudh, SERENA: SchEduling RoutEr Nodes Activity in wireless ad hoc and sensor networks, IWCMC 2008, IEEE International Wireless Communications and Mobile Computing Conference, Crete Island, Greece, August 2008.

[2] Demirkol, C. Ersoy, F. Alagoz, MAC protocols for wireless sensor networks: a survey, IEEE Communications Magazine (2006)

[3] M. Cardei, D. Du, Improving wireless sensor network lifetime through power aware organization, ACM Journal of Wireless Networks, May 2005.

[4] W. Ye, J. Heidemann, and D. Estrin, "An energy-efficient mac protocol for wireless sensor networks," in Proc. IEEE INFOCOM, New York, NY, June 2002, pp. 1567-1576.

[5] G. Lu, B. Krishnamachari, C. Raghavendra, An Adaptive EnergyEfficient and Low-Latency MAC for Data Gathering in Wireless Sensor Networks, Parallel and Distributed Processing Symposium, April 2004.

[6] T. V. Dam, K. Langendoen, An adaptive energy-efficient MAC protocol for wireless sensor networks, ACM SenSys'03, November 2003.

[7] I. Rhee, A. Warrier, L. Xu, Randomized dining philosophers to TDMA scheduling in wireless sensor networks, Technical Report TR-2005-21, Dept of Computer Science, North Carolina State University, April 2005.

[8] V. Rajendran, K. Obraczka, J.J. Garcia-Luna-Aceves, Energy-efficient, collision-free medium access control for wireless sensor networks, Sensys'03, Los Angeles California November 2003.

[9] V. Rajendran, J.J. Garcia-Luna-Aceves, K. Obraczka, Energy-efficient, application-aware medium access for sensor networks, IEEE MASS 2005, Washington, November 2005.

[10] I. Rhee, A. Warrier, M. Aia, J. Min, Z-MAC: a hybrid MAC for wireless sensor networks, SenSys'05, San Diego, California, November 2005

[11] http://www.omnetpp.org/

[12] http://www.inf.usi.ch/postdoc/foerster/downloads.html

[13] http://mobility-fw.sourceforge.net/hp/index.html

[14] Y.R. Faizulkhakov, Synchronization Methods for Wireless Sensor Networks: A Survey, Programming and Computer Software, 33 (4), Plenum Press, 2007. 